H. Kastner, U. Einhorn-Stoll, B. Senge

\title{
New Parameters for the Examination of the Pectin Gelation Process
}

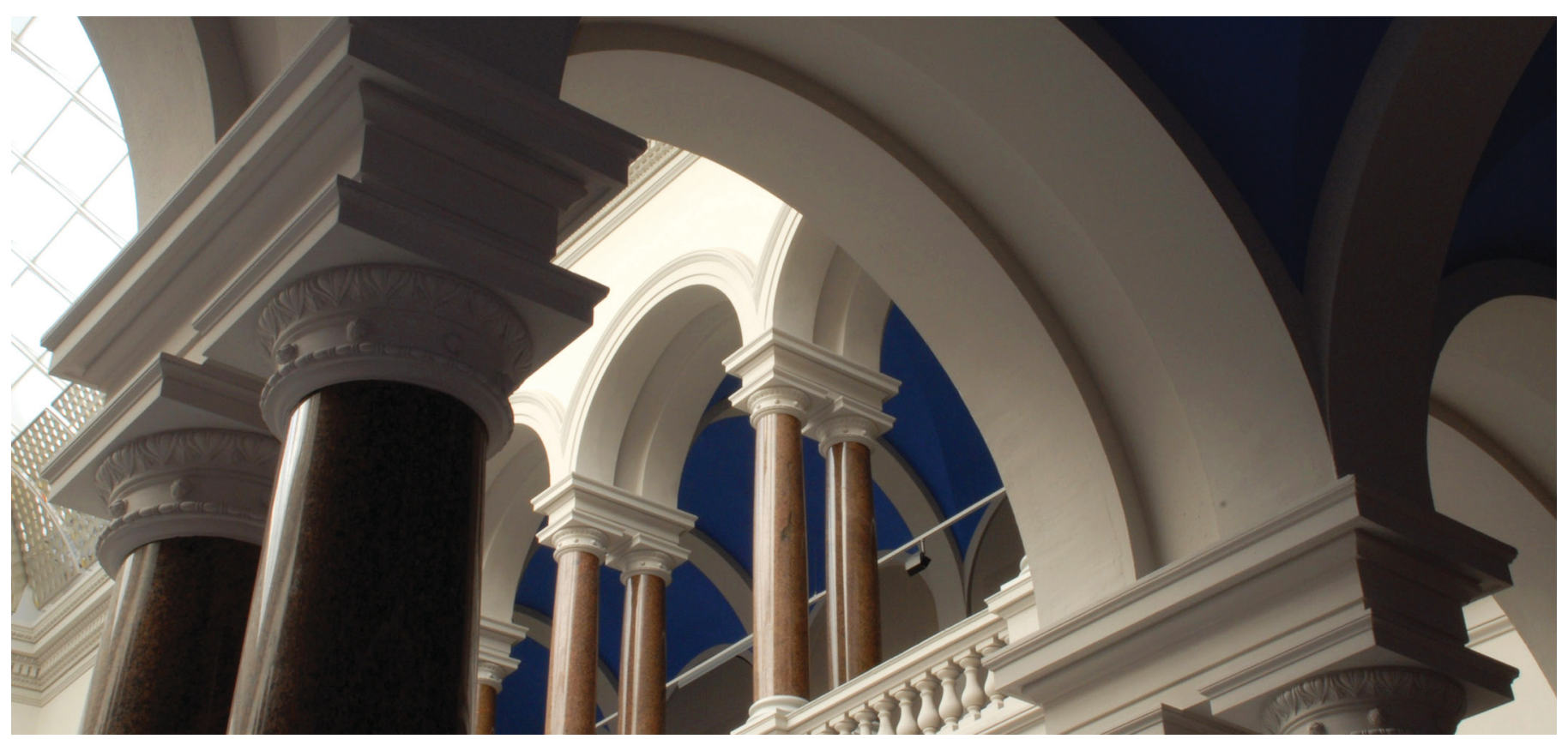

Kastner, H., Einhorn-Stoll, U., \& Senge, B. (2012). New Parameters for the Examination of the Pectin Gelation Process. In Gums and Stabilisers for the Food Industry 16 (pp. 191-197). https://doi.org/10.1039/9781849734554-00191 


\title{
NEW PARAMETERS FOR THE EXAMINATION OF THE PECTIN GELATION PROCESS
}

\author{
H. Kastner, U. Einhorn-Stoll, B. Senge
}

Department of Food Technology and Food Chemistry, Technische Universitaet Berlin, Koenigin-Luise-Strasse 22, D-14195 Berlin, Germany

\section{INTRODUCTION}

Industrial pectins are mainly obtained from by-products of the citrus or apple juice industry by acidic extraction. They are used as gelling or thickening agents in order to improve the texture of food products. Pectins are branched polysaccharides with a backbone of galacturonic acid and neutral sugar side chains, present in the plant cell walls. The galacturonic acid molecules are partly esterified with methanol. Materials with more than 50 $\%$ methoxylated carboxyl groups (degree of methylation DM $>50 \%$ ) are named as highmethoxylated pectins (HMP) and those with less than $50 \%$ are low-methoxylated (LMP). The typical DM for commercial use is about 60 to $77 \%$ for HMP and about 25 to $40 \%$ for LMP. ${ }^{1}$ The DM is crucial for the complex pectin gelation process. HMP form gels in the presence of at least $55 \%$ soluble solids (mostly about $65 \%$ sugar) and at low $\mathrm{pH}(<3.5){ }^{2,3}$ Their gelling mechanism is a combination of hydrophobic interactions between methoxyl groups, favored by higher temperature, and hydrogen bonds between undissociated carboxyl groups, dominating at lower temperature. ${ }^{4}$ The LMP network formation is less dependent on $\mathrm{pH}$ and soluble solids than the HMP gelation, it is promoted by the presence of $\mathrm{Ca}^{2+}$, forming intermolecular ionic junction zones between smooth regions of neighbored chains (egg-box model). ${ }^{1,5}$

Fundamental knowledge of the material and structuring properties of pectins, especially of their gelling behavior, is of crucial importance for their application in foods as well as for the configuration of technological processes. The gelation temperature of pectins depends not only on their botanical source, manufacturing conditions and molecular and material properties but also on the gel preparation procedure and cooling conditions. There are many experimental studies of the complex pectin gelation process as transition from sol to gel as well as of the "gel point", the temperature at which the material properties change from liquid to solid. Sometimes, however, it can be difficult to define a clear gel point because the pectin gelation process is rather complex.

Mechanical properties of final pectin sugar model gels have been investigated for instance using empirical tests, based on gel breaking, or the ${ }^{\circ}$ SAG-method. ${ }^{6,7}$ They are applied in the pectin industry in order to standardize pectins for commercial use. Nowadays mainly fundamental rheological measurements are applied for gel examination. They allow the 
investigation of the structure formation during the gelling process with determination of setting time and temperature (gel point) as well as the final gel properties.

In oscillation measurements, widely used for the examination of the pectin structuring process, the gel point was frequently defined as cross-over of storage modulus $\mathrm{G}^{\prime}$ and loss modulus $G^{\prime \prime}\left(\tan \delta=G^{\prime \prime} / G^{\prime}=1\right)$. This method was developed initially for chemical gelation. ${ }^{8}$ In most food products, however, physical gelation via junction zones occurs and sometimes no clear gel point can be determined for pectin gels, for instance because of pregelation. ${ }^{9,10,11}$ Therefore, a modified method examined the point of intersection as a function of frequency, ${ }^{3,8}$ where the gel point as $\tan \delta=1$ might be defined only in case it is independent on the frequency. ${ }^{11}$ But also this method is not always exact and, therefore, sometimes the described point is named as "apparent gel point". ${ }^{3}$ Other indicators of the gel formation were described by a strong decrease of $\tan \delta^{12,13}$ or using the structure development rate $\mathrm{SDR}=\mathrm{dG}^{\prime} / \mathrm{dt}$ during cooling ${ }^{14}$. Nevertheless, the gel point determination is not completely clear, yet.

The objective of this study is it, therefore, to investigate the structuring process of commercial pectin gels using additional new gelling parameters that allow a detailed examination of influencing factors such as botanical origin, preparation conditions, molecular and material properties, gel preparation (pectin concentration, $\mathrm{pH}$, soluble solid, divalent cations) or experimental parameters like cooling rate on the gelling process.

\section{MATERIALS AND METHODS}

\subsection{Materials}

High or low methylated non-standardized commercial citrus pectins were obtained from three pectin companies. For data protection reasons an anonymous pectin declaration $2 \mathrm{~A}$, $3 \mathrm{~B}$, etc. had to be made. Gelling parameters and the DM of the samples are given in Table 3 , further molecular characteristics and the relating determination methods are presented in a parallel paper. $^{15}$

\subsection{Gel preparation}

The pectin-sucrose-gel composition and preparation was based on the USA-sag method ${ }^{6,7}$ (Table 2). In contrast to HMP gel preparation, the LMP gelation requires $\mathrm{Ca}^{2+}$ and a different $\mathrm{pH}$ (Table 1). All experiments were made at least in duplicate for each pectin gel.

2.2.1 HMP sucrose gel preparation: Dry pectin powder was dissolved in demineralised water. While stirring, the mixture was heated quickly to boiling. The sucrose was added and the HMP-sucrose mass was reduced to a defined value. Afterwards, the $\mathrm{pH}$ of the mass was reduced degraded by addition of tartaric acid solution.

2.2.2 LMP sucrose gel preparation: Demineralised water, citric acid solution and sodium citrate solution were mixed in a steel pot and the dry pectin powder was added while stirring. The mixture was heated quickly to boiling, sucrose was added and the mixture was boiled again. The calcium chloride dehydrate solution was added and while stirring the LMPsucrose mass was reduced to a defined value. The detailed information of the ingredients of the two gels is given in Table 1. 
Table 1 Preparation of gel samples with pectins of different DM

\begin{tabular}{lrr}
\hline Ingredients & HMP gel & LMP gel \\
\hline Pectin content & $2.75 \mathrm{~g}$ & $6.00 \mathrm{~g}$ \\
Demineralized water & $430.00 \mathrm{~g}$ & $637.50 \mathrm{~g}$ \\
Sucrose & $647.25 \mathrm{~g}$ & $264.00 \mathrm{~g}$ \\
$48.8 \%$ tartaric acid solution & $7.00 \mathrm{ml}$ & - \\
$54.3 \%$ citric acid solution & - & $7.50 \mathrm{ml}$ \\
$6 \%$ sodium citrate solution & - & $15.00 \mathrm{ml}$ \\
$2.205 \%$ calcium chloride dehydrate solution & - & $37.50 \mathrm{ml}$ \\
\hline Reduced to & $\mathbf{1 0 1 5} \mathbf{g}$ & $\mathbf{9 0 0 ~ g}$ \\
\hline Total pectin concentration & $\mathbf{0 . 2 7 \%}$ & $\mathbf{0 . 6 7 \%}$ \\
\hline
\end{tabular}

\subsection{Rheological measurements}

Oscillation measurements of pectin-sugar model gels were carried out on a Physica MCR 301 (Anton Paar). The applied geometry was a double gap rotational cylinder CC27/P1 with peltier cylinder temperature system TEZ 150P. After gel preparation, about $15 \mathrm{ml}$ of the sample was transferred immediately into the pre-heated rheometer $\left(105{ }^{\circ} \mathrm{C}\right)$ and cooled to $20{ }^{\circ} \mathrm{C}(\mathrm{HMP})$ or $10{ }^{\circ} \mathrm{C}(\mathrm{LMP})$ with a cooling rate of $1 \mathrm{~K} / \mathrm{min}$. The sample was coated with silicone oil and the cylinder was closed with a special lid in order to avoid evaporation. The dynamic rheological parameters storage modulus $\mathrm{G}^{\prime}$ and loss modulus $\mathrm{G}^{\prime \prime}$ as well as the loss factor $\tan \delta=\mathrm{G}^{\prime \prime} / \mathrm{G}^{\prime}$ of the pectin-sugar model systems were recorded during cooling (temperature sweep) at a frequency of $1 \mathrm{~Hz}$ and deformation amplitude of 0.001 .

\subsection{Ridgelimeter (USA-sag) method}

The USA-sag method was implemented by the IFT committee for pectin standardisation. This method is rather empirical but frequently used for routine or quality tests in the pectin industry, yet.

The rest of the hot pectin-sugar solution, prepared as described above, was filled into three special glasses and stored at $25^{\circ} \mathrm{C}$ for $24 \mathrm{~h}$ before measurement. For an accurate result, the average gel properties of the three gel cones had to be within the limits shown in Table 2.

A Lab850 pH-meter with a special penetration electrode from Schott Instruments was used for the determination of the $\mathrm{pH}$ in the gels after Ridgelimeter measurements. Moreover, the soluble solid SS was determined in the gel using a refractometer (Schmidt and Haensch).

Table 2 Conditions for the different pectin gels

\begin{tabular}{lcc}
\hline Ingredients & HMP gel & LMP gel \\
\hline $\mathrm{pH}$ & $2.2-2.4$ & $2.8-3.2$ \\
$\mathrm{SS}$ & $64.5-65.5 \%$ & $30-32 \%$ \\
Gel strength & \multicolumn{2}{c}{$19.5-27.0 \% \mathrm{sag}$} \\
\hline
\end{tabular}




\subsection{Examination of structure formation}

Three characteristic gelling temperatures were calculated: The (apparent) gel point temperature $(G P)$ as cross-over of $\mathrm{G}^{\prime}$ and $\mathrm{G}^{\prime \prime}$, the initial structuring temperature (IST) and critical structuring temperature (CST) as shown in Figure 1 from the first derivation of the storage modulus $\left(\mathrm{dG}^{\prime} / \mathrm{dt}=\right.$ structure formation velocity) using Origin 8.1 software. The IST is defined as the temperature at which the structure formation velocity was different from 0 for the first time. The CST is the extrapolated temperature for the first strong increase of the structure formation velocity. In contrast to the GP, these two structuring temperatures could be detected for any pectin we have ever investigated. Moreover, from the structure formation velocity curve often different structuring phases could be identified.

The details of the new defined parameters are shown in Figure 1.

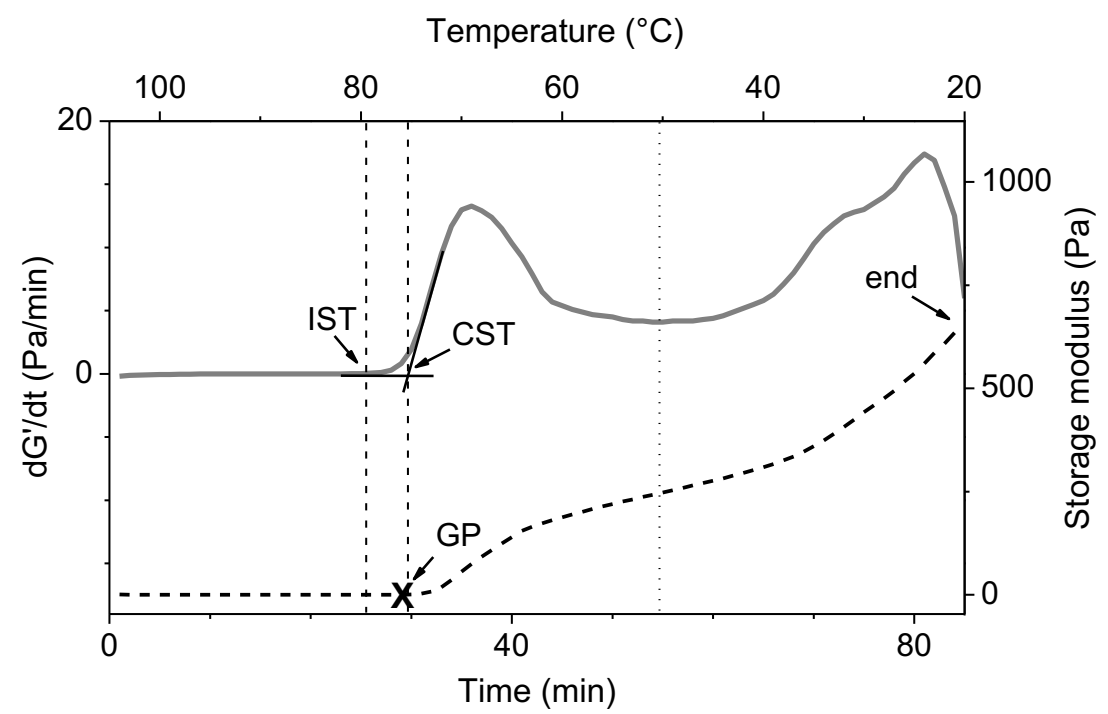

Figure 1 Evaluation of the structure formation during cooling of an HMP-gel (sample $3 A)$. Full line $=d G^{\prime} / d t ;$ dotted line $=$ storage modulus $G^{\prime} ;$ IST $=$ initial structuring temperature; $C S T=$ critical structuring temperature; end $=$ end level at $20^{\circ} \mathrm{C}$; GP = gel point $=\tan \delta=1$.

\section{RESULTS AND DISCUSSION}

\subsection{Structure formation temperatures}

The rheological studies were made using different but constant preparation conditions for HMP and LMP, respectively, (Table 1) and allowed the comparison of the gelling processes and gel properties. Sometimes, the rheological measurements gave no clear setting point as intercept of $\mathrm{G}^{\prime}$ and $\mathrm{G}^{\prime \prime}$, causing uncertainty for identifying the exact gelling temperature. Especially in these cases, the new structuring parameters IST and CST allowed a better description and comparison of the structure formation process.

The structure formation velocity curve $\mathrm{dG}^{\prime} / \mathrm{dt}$ characterised not only the gelling kinetic but also different phases in the gelation process by alterations of the structure formation velocity. 
Table 3 Characteristics and gelling properties of the tested pectin samples.

$D M=$ degree of methoxylation, $G P=$ gelling point, $I S T=$ initial structuring temperature, CST = critical structuring temperature, $G^{\prime}$ end $=$ final storage modulus, tan $\delta_{\text {end }}=$ final loss factor .

\begin{tabular}{cccccccc}
\hline & & & \multicolumn{5}{c}{ Parameters of the gelation process } \\
\cline { 4 - 8 } Source & Sample & $\begin{array}{c}D M \\
\%\end{array}$ & $\begin{array}{r}G P \\
{ }^{\circ} C\end{array}$ & $\begin{array}{c}I S T \\
{ }^{\circ} C\end{array}$ & $\begin{array}{c}C S T \\
{ }^{\circ} C\end{array}$ & $\begin{array}{c}G^{\prime} \text { end } \\
P a\end{array}$ & tan $\delta_{\text {end }}$ \\
\hline $\mathbf{1}$ & 1B & 59.6 & 93 & 81 & 91 & 1077 & 0.078 \\
& 1C & 24.2 & 51 & 42 & 53 & 91 & 0.151 \\
\hline $\mathbf{2}$ & 2A & 68.9 & 86 & 88 & 85 & 815 & 0.068 \\
& 2B & 55.1 & 58 & 62 & 58 & 639 & 0.054 \\
& 2C & 30.1 & 43 & 51 & 37 & 81 & 0.200 \\
\hline $\mathbf{3}$ & 3A & 69.8 & 77 & 79 & 76 & 587 & 0.061 \\
& 3B & 57.1 & 57 & 60 & 56 & 877 & 0.051 \\
& 3C & 63.6 & 67 & 70 & 66 & 788 & 0.057 \\
& 3D & 32.8 & 43 & 60 & 31 & 44 & 0.166 \\
& 3E & 30.2 & 58 & 66 & 55 & 128 & 0.128 \\
& 3F & 27.7 & - & 79 & 69 & 293 & 0.120 \\
& 3G & 69.0 & 70 & 72 & 69 & 296 & 0.090 \\
& 3H & 56.5 & 54 & 54 & 53 & 348 & 0.087 \\
\hline
\end{tabular}

\subsection{Gelling process}

A higher DM, in general, leaded to a faster start of the incipient structuring process at higher gelation temperatures (Table 3 and Figure 2a). This is well-known and crucial for the pectin application.

In order to investigate the influence of manufacturing conditions on the gelling process, two HMP (2A and 3A) and two LMP (2C and 3E), respectively, with similar DM but from different companies were compared. Differences in the start of the structuring process and structuring velocity were observed (Figure $2 b$ and $2 c$, Table 3 ). As shown in Figure $2 b$, there was an earlier increase in $\mathrm{dG}^{\prime} / \mathrm{dt}$ of the $2 \mathrm{~A}$ gel, with structure formation temperatures GP, IST and CST about $9 \mathrm{~K}$ higher than in the 3A gel. The same was found for the two LMP with a difference $>15 \mathrm{~K}$. This means that the gelling properties of pectins were strongly dependent on their processing conditions and that the DM is one indicator but not the only parameter for evaluating the gelling properties.

An increase or decrease of structure formation velocity $\mathrm{dG}^{\prime} / \mathrm{dt}$ can indicate different gelling process phases. It seems that single structuring mechanisms (hydrogen bonds beside hydrophobic interaction for HMP gelation, egg-box junction zones via calcium bridges and hydrogen bonds for LMP) occur in typical temperature ranges of the gelling process. It was found that, even if the IST for two tested HMP or two LMP varied, the typical structuring phases during cooling can behave comparable. Also using the SDR theory ${ }^{14}$, two phases of the gelation process have been already described in past publications. The publication of further results in this field with other influencing parameters is in preparation. 

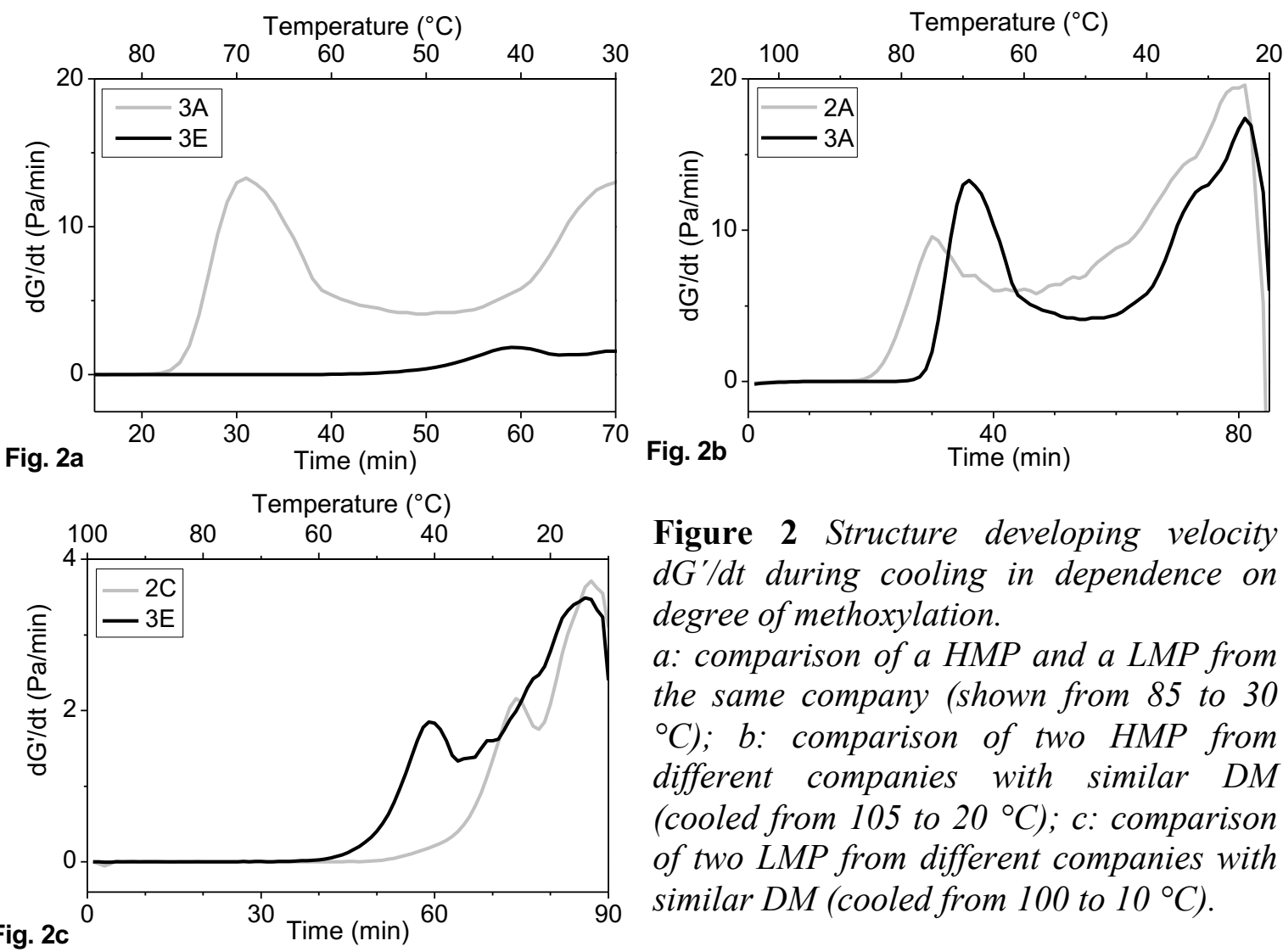

Figure 2 Structure developing velocity $d G^{\prime} / d t$ during cooling in dependence on degree of methoxylation.

a: comparison of a HMP and a LMP from the same company (shown from 85 to 30 $\left.{ }^{\circ} \mathrm{C}\right)$; $b$ : comparison of two HMP from different companies with similar DM (cooled from 105 to $20^{\circ} \mathrm{C}$ ); c: comparison of two LMP from different companies with similar DM (cooled from 100 to $10^{\circ} \mathrm{C}$ ).

The relationship between CST and GP of the tested gels is shown in Figure 3. From this significant correlation can be concluded that the newly defined structuring temperatures CST and the nearly CST - parallel IST are a complementary way to evaluate the incipient gelling process, especially for gels without a clear $G^{\prime}-G^{\prime \prime}$ cross-over. The influence of the manufacturing conditions on the setting behavior of pectins could be sufficiently examined by IST and CST.

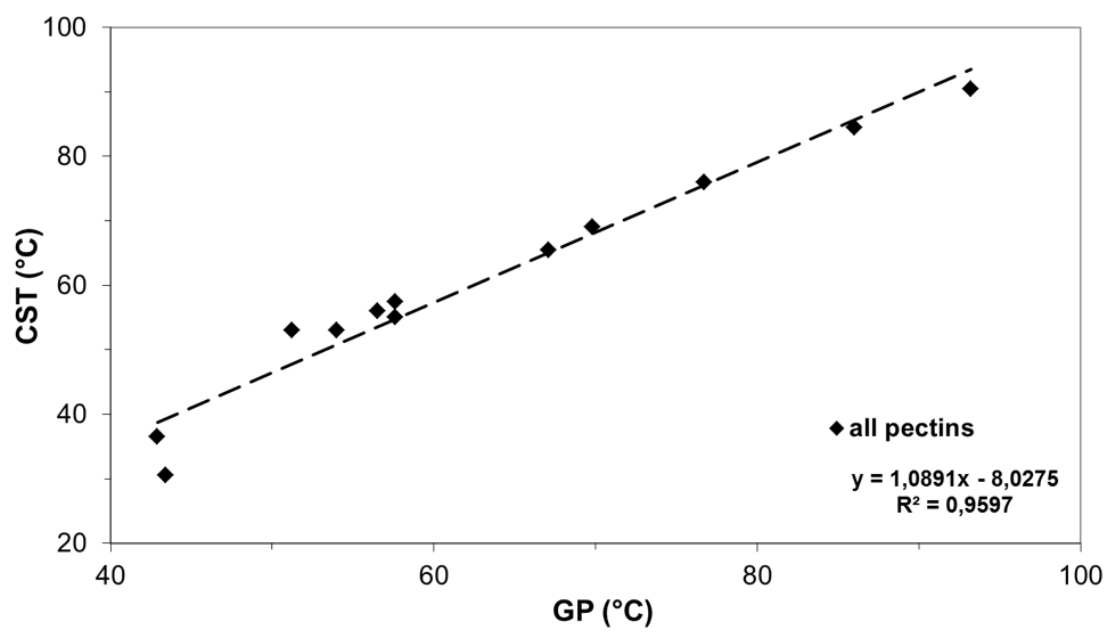

Figure 3 Correlation of structure formation temperatures GP and CST for all pectin sucrose gels. 


\section{CONCLUSIONS}

The analysis of the chemical structure of the examined pectins from different companies showed the complexity of the polymer properties and their influence on the pectin gel characteristics, depending on the pectin manufacturing process. The newly defined initial structuring temperature IST and critical structuring temperature CST were, beside the GP, suitable parameters in order to describe the incipient gel structuring process for both types of pectins. Even samples with no clear GP could be evaluated this way. Therefore, IST and CST have proved to be valuable complementary parameters and can help to understand the structure formation processes more detailed. They can be used for optimizing the pectin production process as well as the pectin application in food products.

\section{References}

1 A.G.J. Voragen, W. Pilnik, J.-F. Thibault, M.A.V. Axelos and C.M.G. Renard, 'Pectins' in Food Polysaccharides and Their Applications, eds., A.M. Stephen, New York, Marcel Dekker Inc., 1995, pp. 287-339.

2 B.R. Thakur, R.K. Sing and A.K. Handa, Crit. Rev. Food Sci. Nutr., 1997, 37, 7.

3 J.A. Lopes da Silva, M.P. Goncalves and M.A. Rao, Int. J. Biological Macromolecules, 1995, 17, 25.

4 D. Oakenfull and A. Scott, J. Food Sci., 1984, 49, 1093.

5 E.R. Morris, D.A. Powell, M.J. Gidley and D.A. Rees, J. Molecular Biology, 1982, 155, 507.

6 IFT Committee, Food Technology, 1959, 8, 496.

7 R.E. Cox and R.H. Higby, Food Industry, 1944, 16, 441.

8 H.H. Winter and F. Chambon, Journal of Rheology, 1986, 30, 367.

9 D. Lootens, F. Capel, D. Durand, T. Nicolai, P. Boulenguer and V. Langendorff, Food Hydrocolloids, 2003, 17, 237.

10 A. Slavov, C. Garnier, M.-J. Crepeau, S. Durand, J.-F. Thibault and E. Bonnin, Carbohydrate Polymers, 2009, 7, 876.

11 P. Stang-Holst, A.-L. Kjöniksen, H. Bu, S.-A. Sande and B. Nyström, Polymer Bulletin, 2006, 56, 239.

12 A. Dahme, J. Texture Studies, 1992, 23, 1.

13 S. Neidhart, C. Hannak and K. Gierschner, 'Sol-gel transitions of high-esterified pectins and their molecular structure' in Advances in Pectin and Pectinase Research, eds., A.G.J. Voragen, H. Schols and R. Visser, Kluwer, 2003, pp. 431-448.

14 J.-T. Fu and M.A. Rao, Food Hydrocolloids, 2001, 15, 93.

15 U. Einhorn-Stoll, H. Kastner and B. Senge, Gums and stabilisers for the food industry, 2011, 16. 